\title{
Extreme Ductility in Freestanding Polystyrene Thin Films
}

\author{
Nathan R. Velez ${ }^{*, 1,2}$, Frances I. Allen ${ }^{1,2}$, Mary Ann Jones ${ }^{3}$, Sanjay \\ Govindjee $^{4}$, Gregory F. Meyers ${ }^{3}$, and Andrew M. Minor ${ }^{*, 1,2}$ \\ ${ }^{1}$ Department of Materials Science and Engineering, UC Berkeley, Berkeley, CA \\ ${ }^{2}$ National Center for Electron Microscopy, Molecular Foundry, LBNL, Berkeley, CA \\ ${ }^{3}$ Core R\&D - Analytical Sciences, The Dow Chemical Company, Midland, MI \\ ${ }^{4}$ Department of Civil and Environmental Engineering, UC Berkeley, Berkeley, CA
}

\section{Supplementary Experimental Figures}
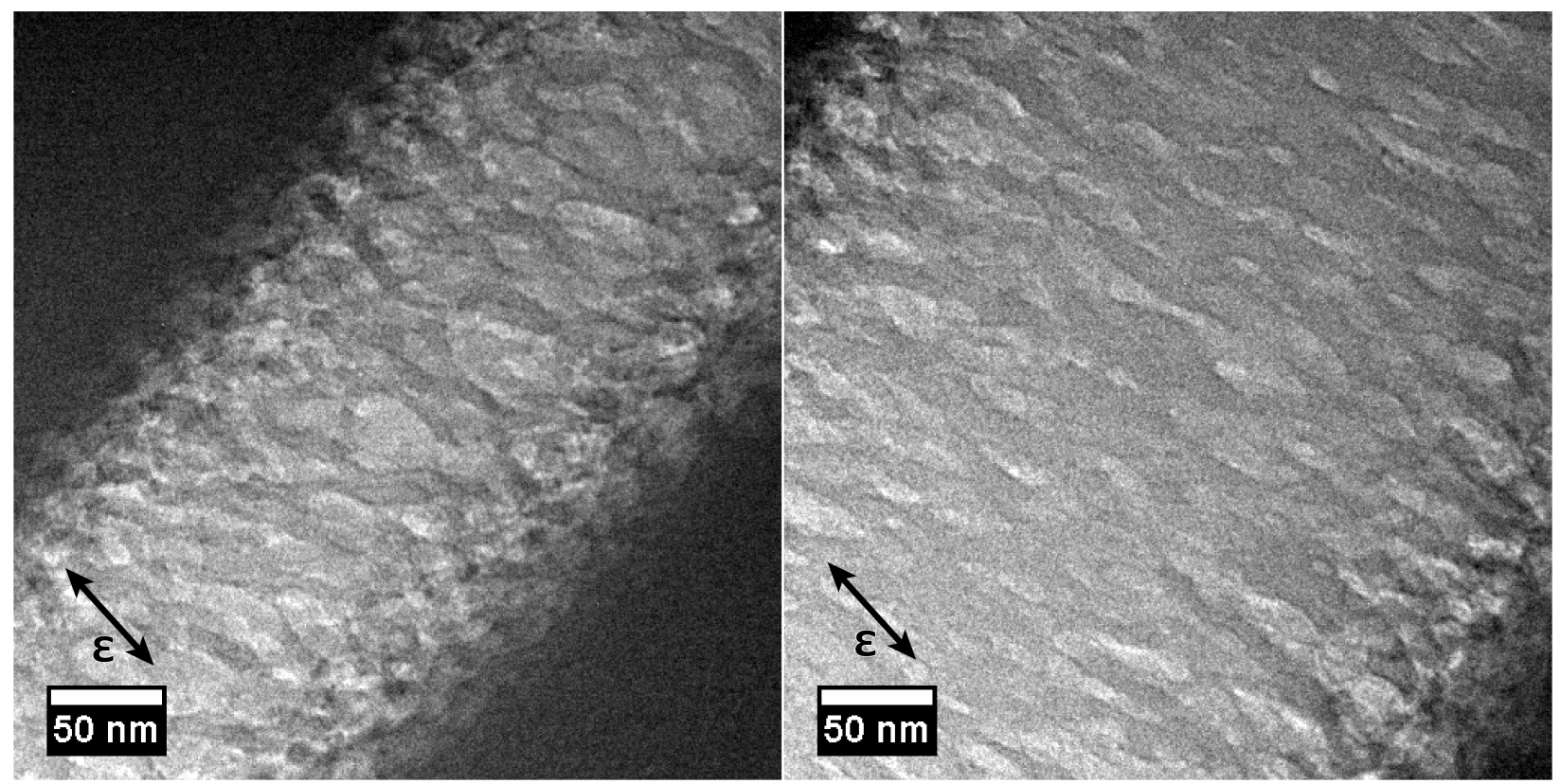

Supplemental Figure 1: Two TEM images of an annealed thin film of polystyrene displaying the 2D crazing morphology known as "perforated sheets," which results from the absence of out-of-plane stress. (Thickness $=\sim 250 \mathrm{~nm}$; arrows indicate the direction of strain.) 


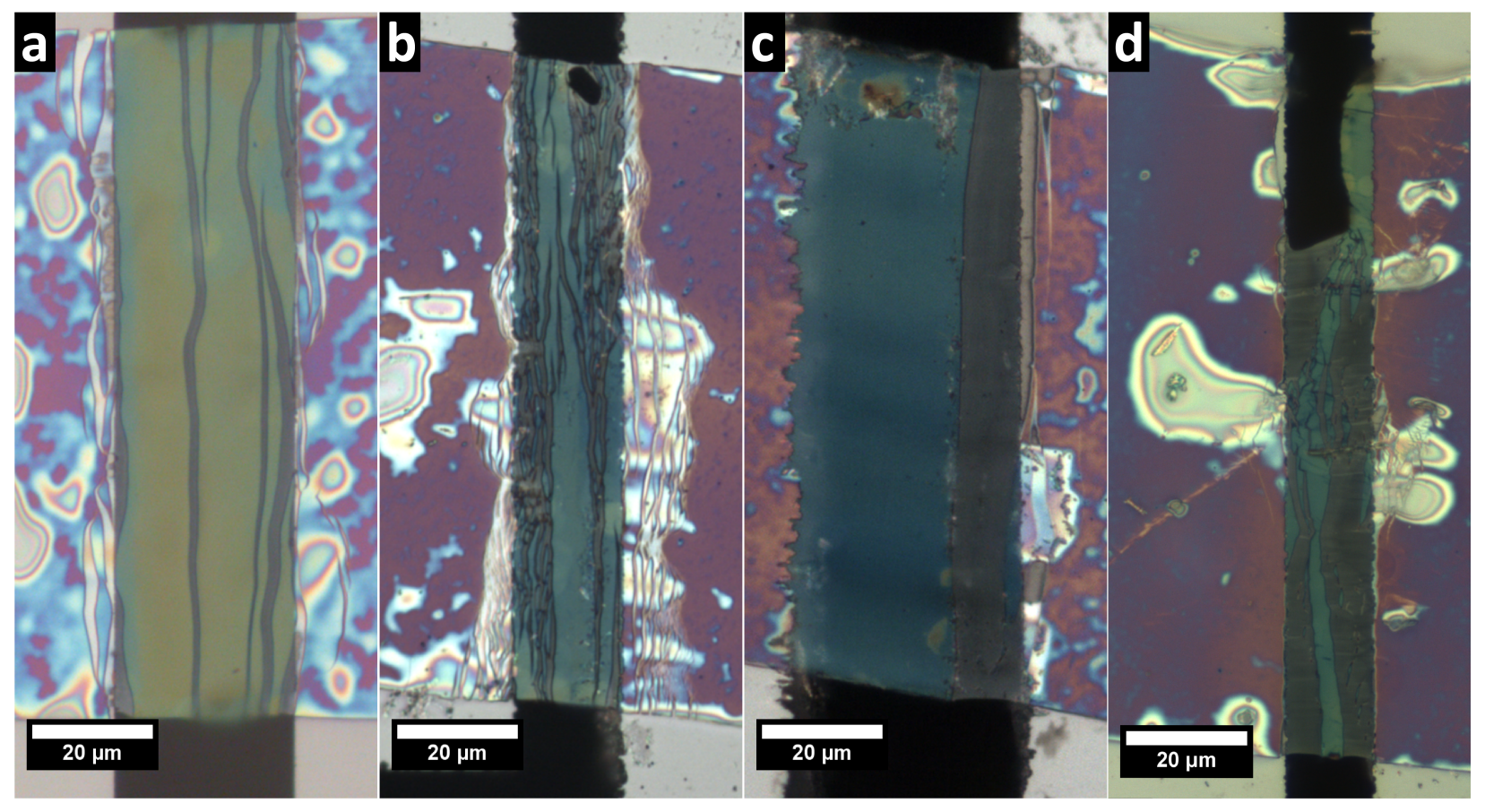

Supplemental Figure 2: Correlating optical images of polystyrene thin films shown in Figure 3. a) Annealed, $270 \mathrm{~nm}$ thick film strained to 50\%. b) Annealed, $240 \mathrm{~nm}$ thick film strained to $\sim 150 \%$, oriented such that the tensile direction was perpendicular to the cutting direction. c) Unannealed, $250 \mathrm{~nm}$ thick film strained to 50\%. d) Unannealed, 260 $\mathrm{nm}$ thick film strained to $\sim 275 \%$. Notch was introduced at top edge during deposition, but only propagated partially across the film upon straining. Initial tensile gaps in (a-d) were $20,7,25$, and $4 \mu \mathrm{m}$, respectively. 


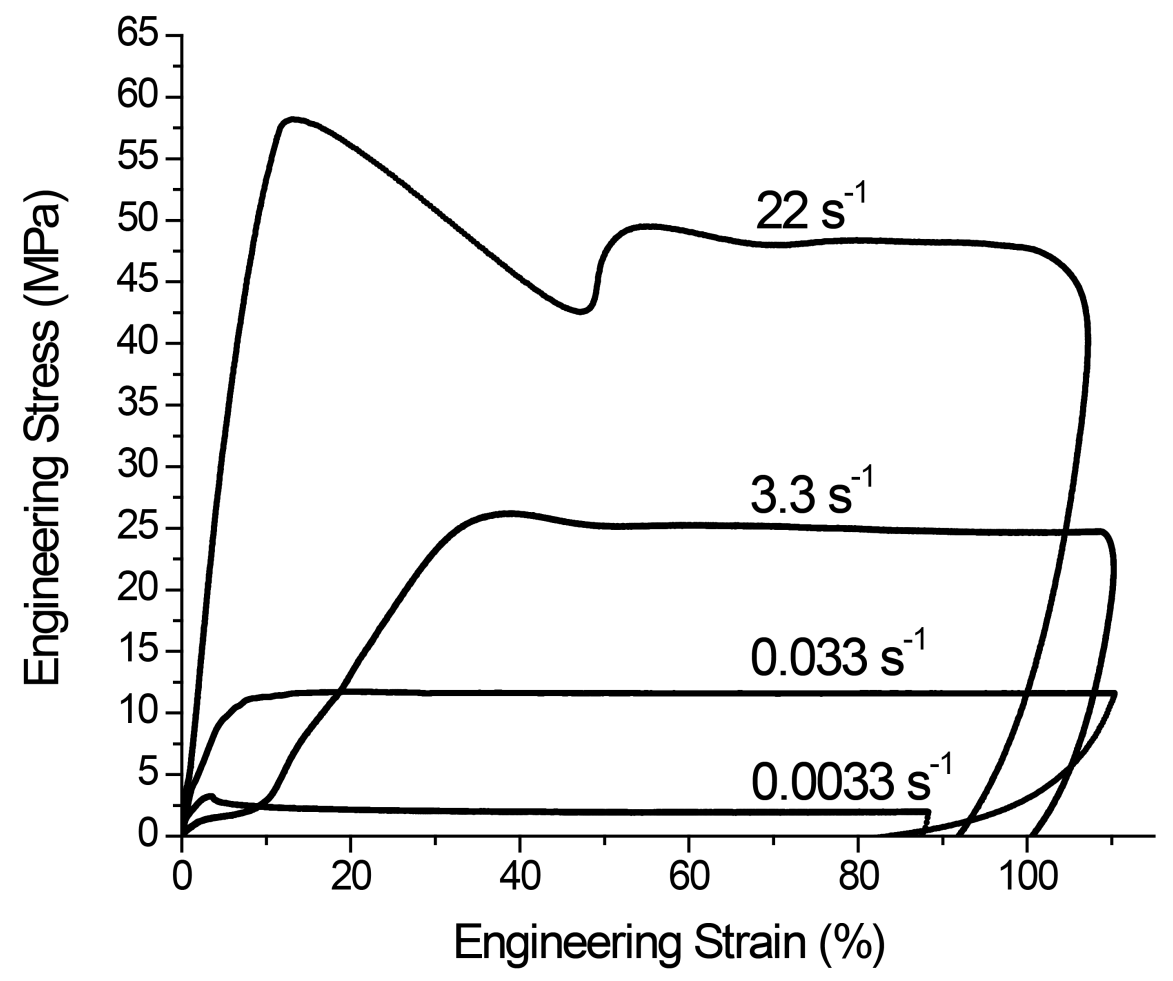

Supplemental Figure 3: Stress-strain curves obtained at different strain rates (indicated above each curve). From top to bottom, the film thicknesses are: 290, 240, 450, and 340 nm. The negative unloading segments are omitted for clarity. 

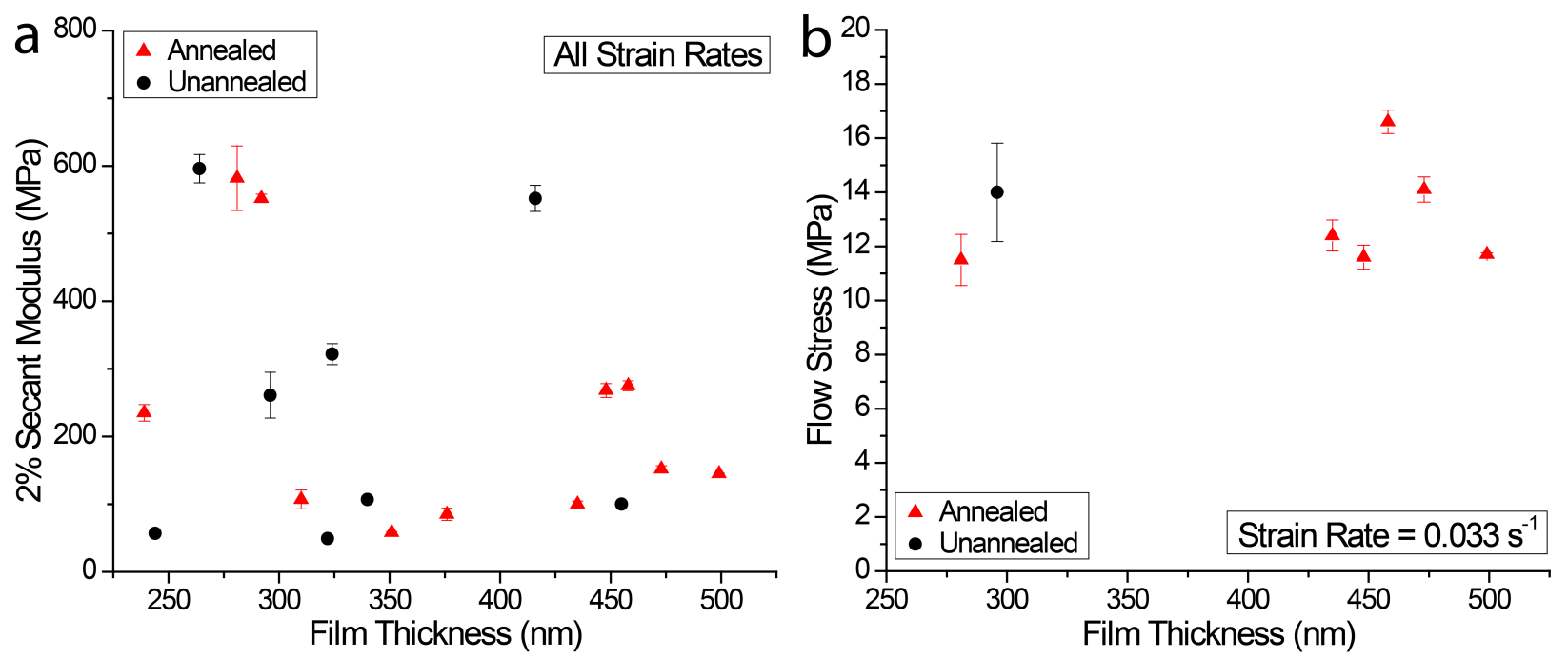

Supplemental Figure 4: a) Plot of the $2 \%$ secant modulus with respect to film thickness for all strain rates. While the values are an order of magnitude lower than for bulk polystyrene ( $\sim 3 \mathrm{GPa})$, there does not appear to be any dependence on annealing or on film thickness in the range tested. b) Plot of the flow stress (strongly strain-rate dependent) with respect to film thickness for experiments performed at a strain rate of $0.033 \mathrm{~s}^{-1}$. Similar to the $2 \%$ secant modulus, the flow stress does not appear to exhibit any thickness dependence. (Error bars derived from the standard deviation between thickness measurements acquired from several SPM images taken at different regions of the film.) 

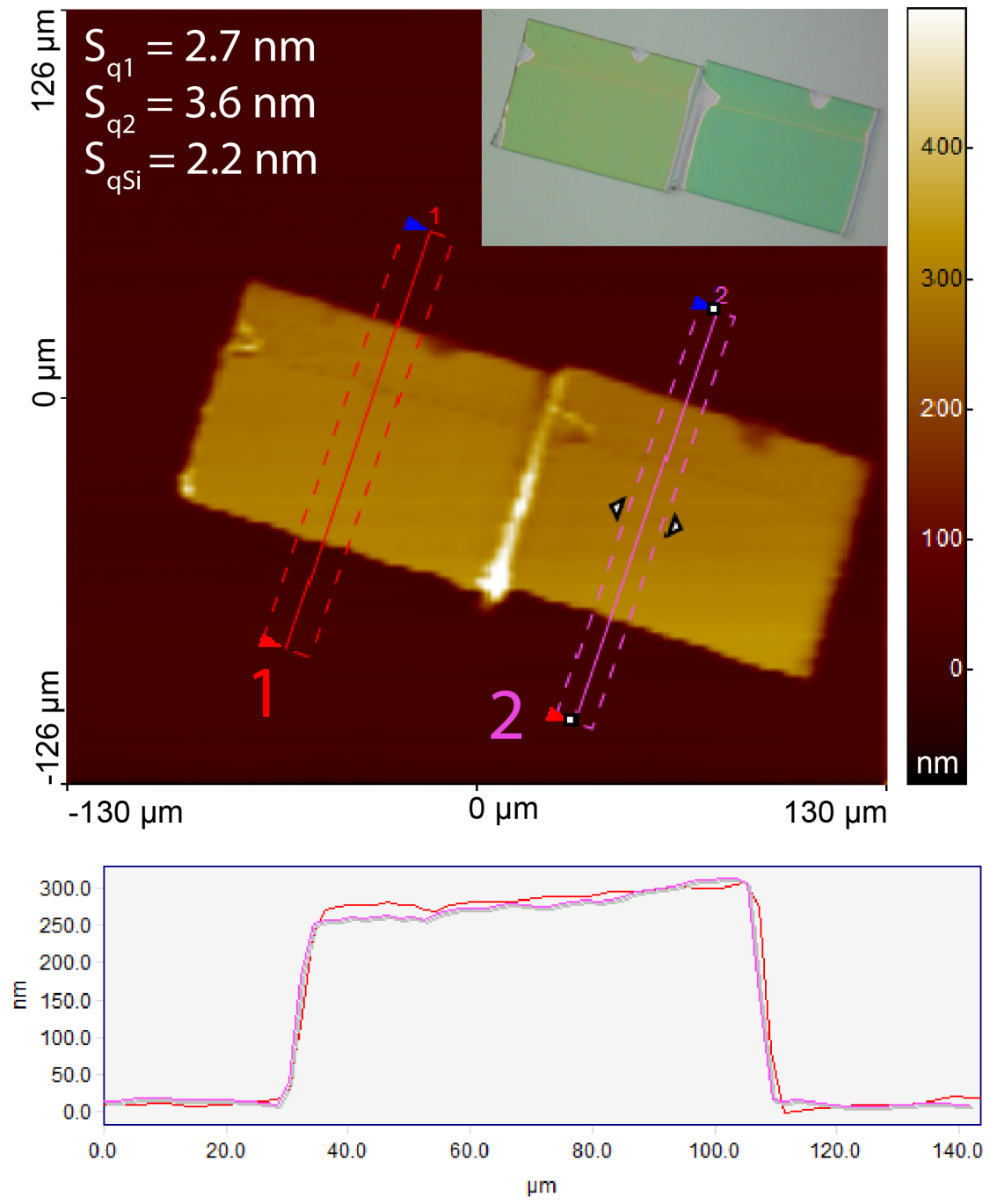

Supplemental Figure 5: TOP: Topography map of two microtomed thin films of polystyrene deposited onto a silicon wafer obtained with a KLA Tencor P17 stylus profilometer. The RMS surface roughness, Sq, was calculated for each film, using the region of interest indicated $\left(\mathrm{S}_{\mathrm{q} 1}=2.7 \mathrm{~nm}\right.$ and $\left.\mathrm{S}_{\mathrm{q} 2}=3.6 \mathrm{~nm}\right)$, as well as the silicon substrate $\left(\mathrm{S}_{\mathrm{qSi}}=\right.$ $2.2 \mathrm{~nm})$. An optical image of the films is shown in the inset. BOTTOM: Line profiles of the films indicated by the solid line for each region of interest. 


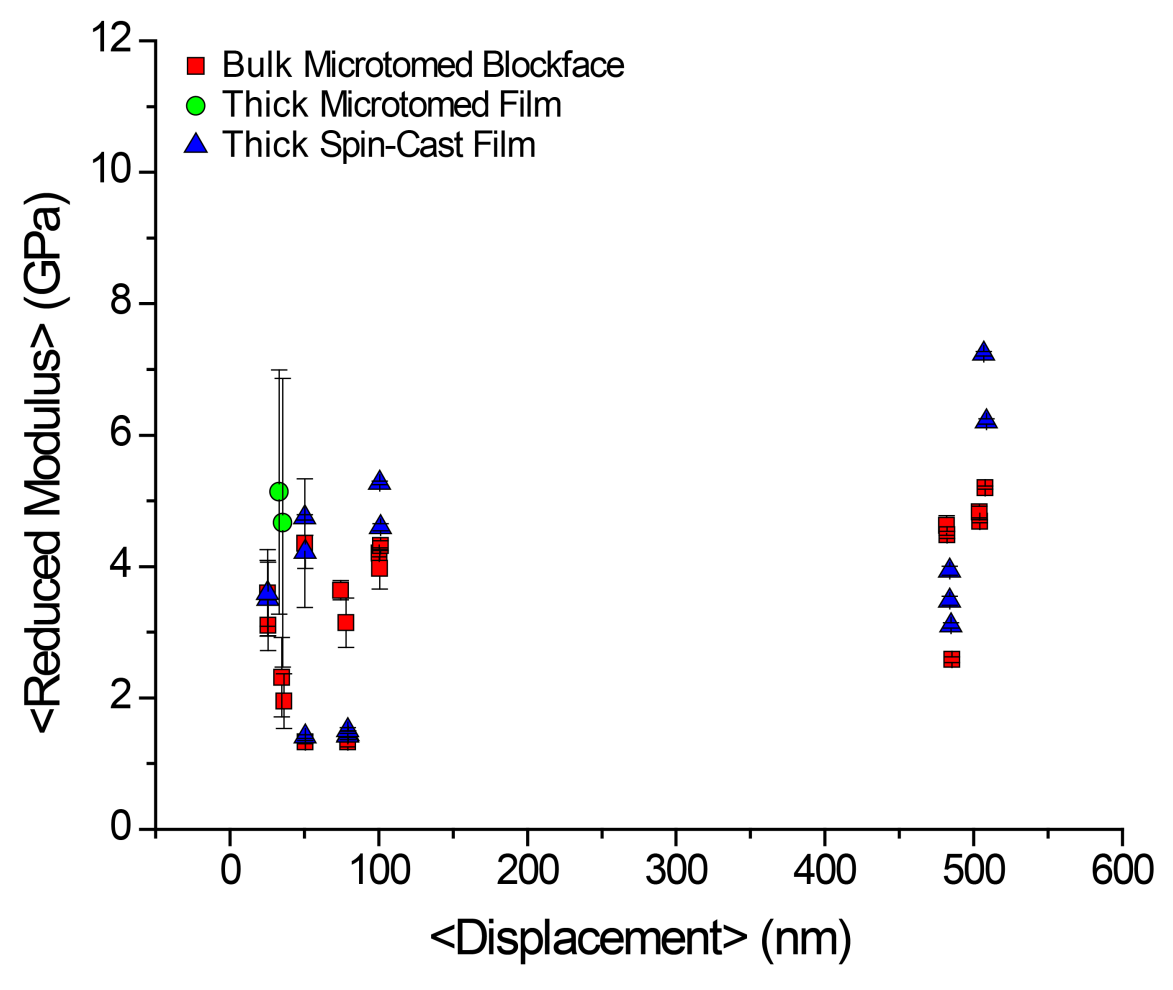

Supplemental Figure 6: Nanoindentation data for the bulk microtomed blockface, thick $(\sim 1 \mu \mathrm{m})$ microtomed films, and thick $(\sim 1 \mu \mathrm{m})$ spin-cast films. No significant difference between the sample preparation types is observed and the values are near the expected value of $3 \mathrm{GPa}$. Each datapoint represents 25 indents.
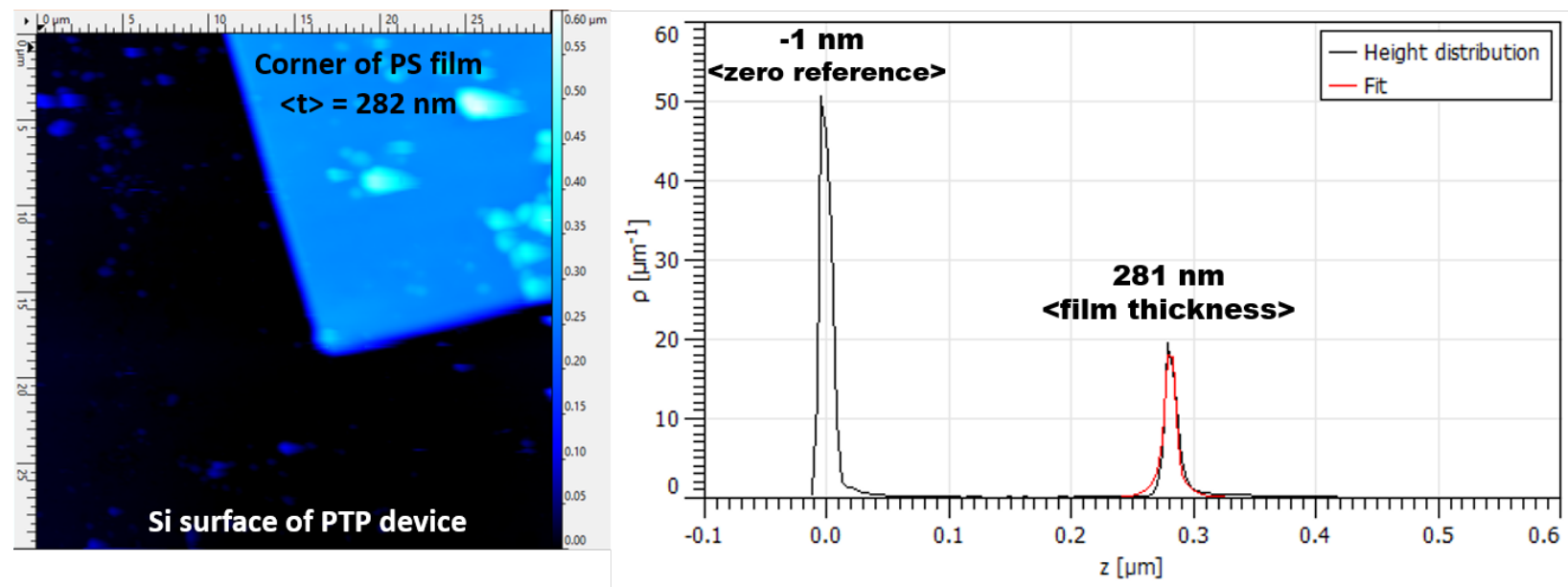

Supplemental Figure 7: LEFT) 30x30 $\mu \mathrm{m}$ topography image showing the corner of a polystyrene thin film (blue) deposited on a PTP device (black). Color scale indicates zheight, ranging from 0 to $600 \mathrm{~nm}$. RIGHT) Histogram of $z$-height values where the positions of the film and substrate peaks (indicated) were determined by fitting a Gaussian curve (red line shows the Gaussian fit for the film height). Gwyddion (v2.52) software was used for all SPM analysis. 


\section{Linear Elastic Simulation of a Thin Film in Planar Tension}

The basic deformation state of the specimen in the Push-to-Pull (PTP) device is one of planestrain tension. Assuming that the $x$-coordinate direction aligns with the opening direction of the PTP device, the $y$-direction aligns with the through-thickness sample direction, and the $z$-direction aligns with the width direction of the sample, then the specimen experiences (predominantly) a tensile stress in the $x$-direction, and an induced tension (due to a Poisson effect) in the $z$-direction. The specimen is largely stress free in the $y$-direction. Shear stresses are largely absent.

There are, however, grip effects which induce local stress concentrations and artifacts associated with the asymmetric (bottom only) gripping. As noted in [2] when a free surface is sharply clamped a stress singularity is induced at the location where the displacements are first restrained; in the case of linear elasticity this singularity has the same order as that at the tip of a sharp crack $O(1 / \sqrt{r})$. Surprisingly, the exceedingly high ductilities that we observe are robust even in the presence of these "crack-like" stress concentrations.

To better elucidate the deformation state of the specimen, we have performed threedimensional linear elastic finite element computations to highlight the deformation state in the PTP. This gives a fuller picture of the tests performed. The computation were run using the general purpose finite element code FEAP [1]. The material was modeled using linear elastic 27-noded tri-quadratic brick elements in quarter-symmetry. In the quartersymmetric model there were 8 nodes in the $y$-direction, 72 nodes in the $x$-direction for the freestanding part of the film, 32 nodes in the $x$-direction over the grip, and 80 nodes in the $z$-direction. The resulting quarter-symmetry model consisted of 687,834 nodes and 74,880 elements, giving a total of 1,965,255 equations after accounting for boundary conditions. To simulate the grips, the nodes on the underside of the specimen were fixed and displaced in the $x$-direction.

Linear elasticity was used to model the material since to-date there are no well accepted constitutive models for the behavior we have seen in the experiments. Notwithstanding, linear elasticity still allows one to gain a sound understanding of the deformation state during the test. For examples run the Young's modulus was $E=3.5 \mathrm{GPa}$ and the Poisson ratio was $\nu=0.35$. The film thickness $t=250 \mathrm{~nm}$, the full-width $w=70 \mu \mathrm{m}$, full-span $s=4.5 \mu \mathrm{m}$, and the grip overhang $g=1 \mu \mathrm{m}$ - a distance sufficient to eliminate any freeedge effects. In all results shown, the grips were displaced so as to induce a strain in the $x$-direction of approximately 1.42 mstrain.

\section{Plane-strain nature of deformation}

Supplemental Figure 8 demonstrates the plane strain nature of the deformation of the specimen. The view shown is a top-down look and the contours correspond to the $z$-displacement of the specimen. Note that the $z$-displacement is largely zero, with a small amount of pull-in at the free edges, top and bottom. 


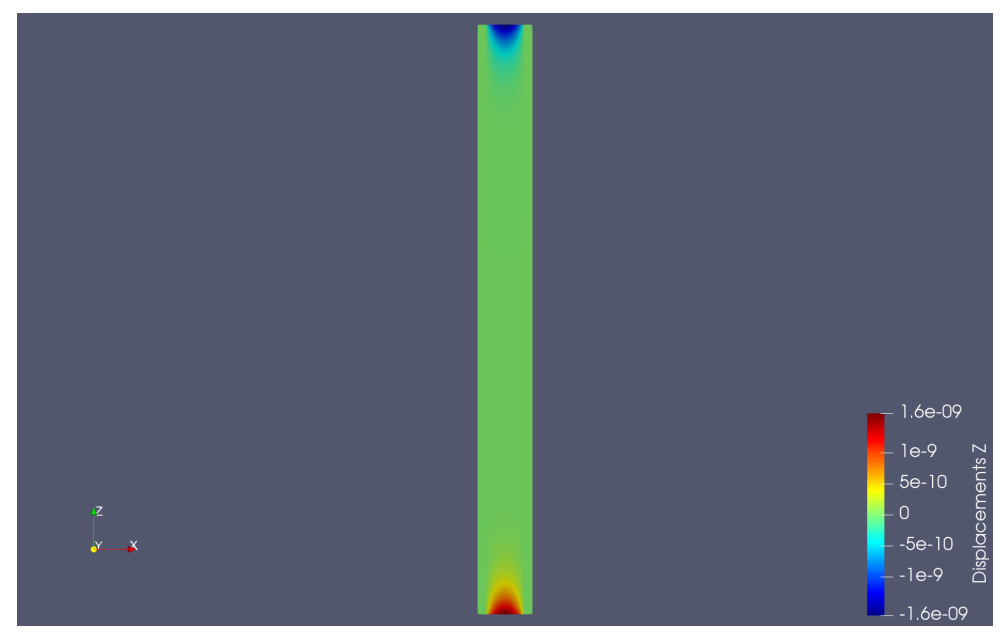

Supplemental Figure 8: $z$-displacement in the test film (linear elastic simulation) at an opening strain of approximately 1.42 mstrain. Displacement in meters.

\section{Opening stress}

Supplemental Figures 9 and 10 show the uniformity of the $x$-direction stress in the specimen, giving a top and bottom view, respectively. It is seen that the stress field in the freestanding portion is very uniform. The dark blue stripes are the portions of the film resting on the grip. When viewed from below (Supplemental Figure 10) the grip edge is sharply delineated. From the top there is a slight diffusion indicating a slight through-thickness effect from the grip geometry. This effect can be seen more clearly in Supplemental Figure 11 which shows a cross-sectional view of the $x$-direction stress at the mid-plane of the full specimen. Note also the stress concentration on the bottom edge due to the grip, which can also be see in the cross-sectional plot of the Mises stress in Supplemental Figure 12 - observe the "red" hot-spots. 


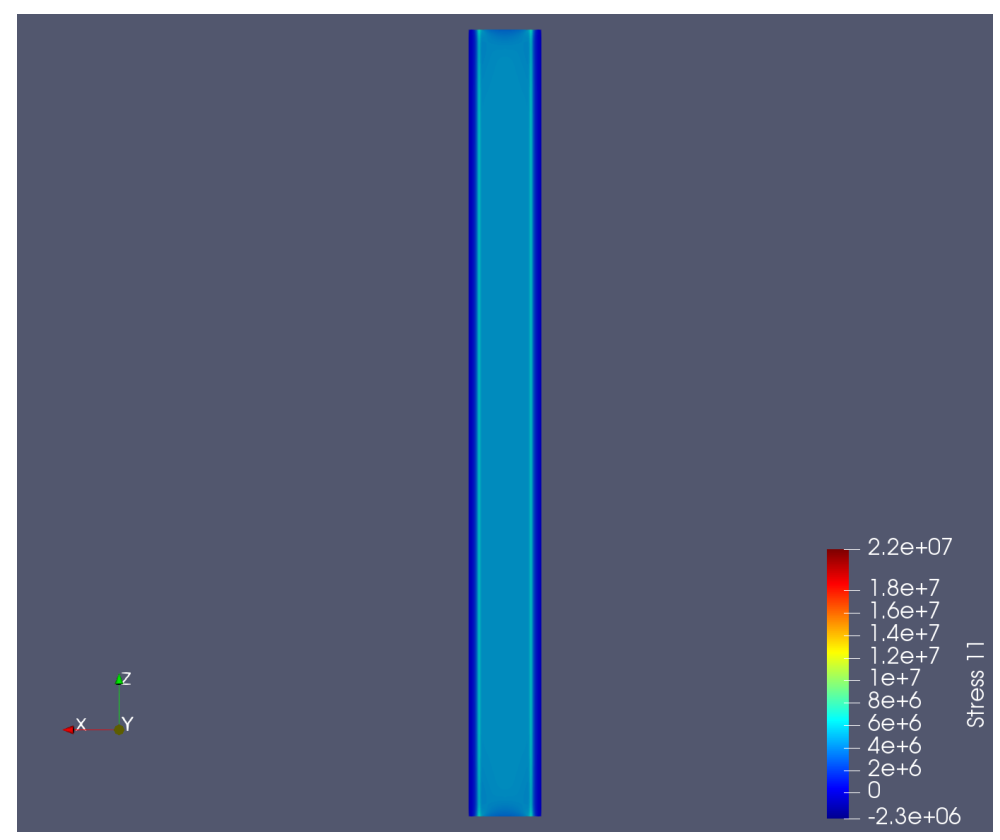

Supplemental Figure 9: $x$-direction stress in the test film (linear elastic simulation) at an opening strain of approximately 1.42 mstrain; top view. Stress in Pascals.

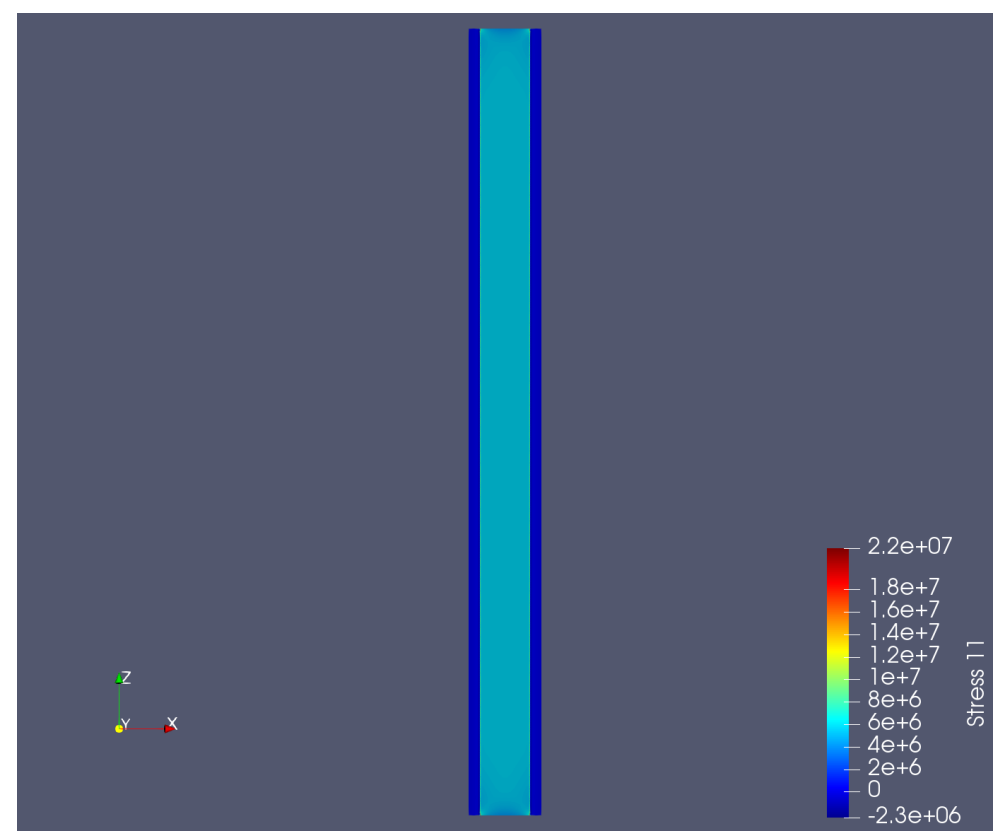

Supplemental Figure 10: $x$-direction stress in the test film (linear elastic simulation) at an opening strain of approximately 1.42 mstrain; bottom view. Stress in Pascals. 


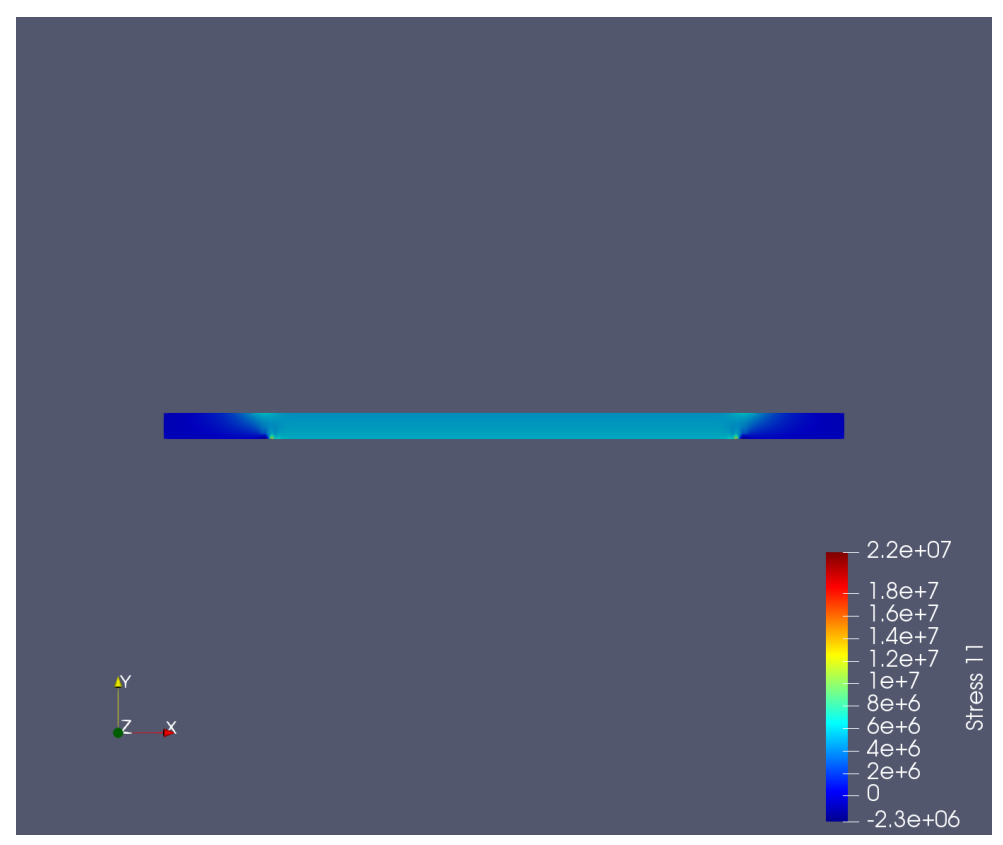

Supplemental Figure 11: $x$-direction stress in the test film at mid-span (linear elastic simulation) at an opening strain of approximately 1.42 mstrain; cross-sectional view. Stress in Pascals.

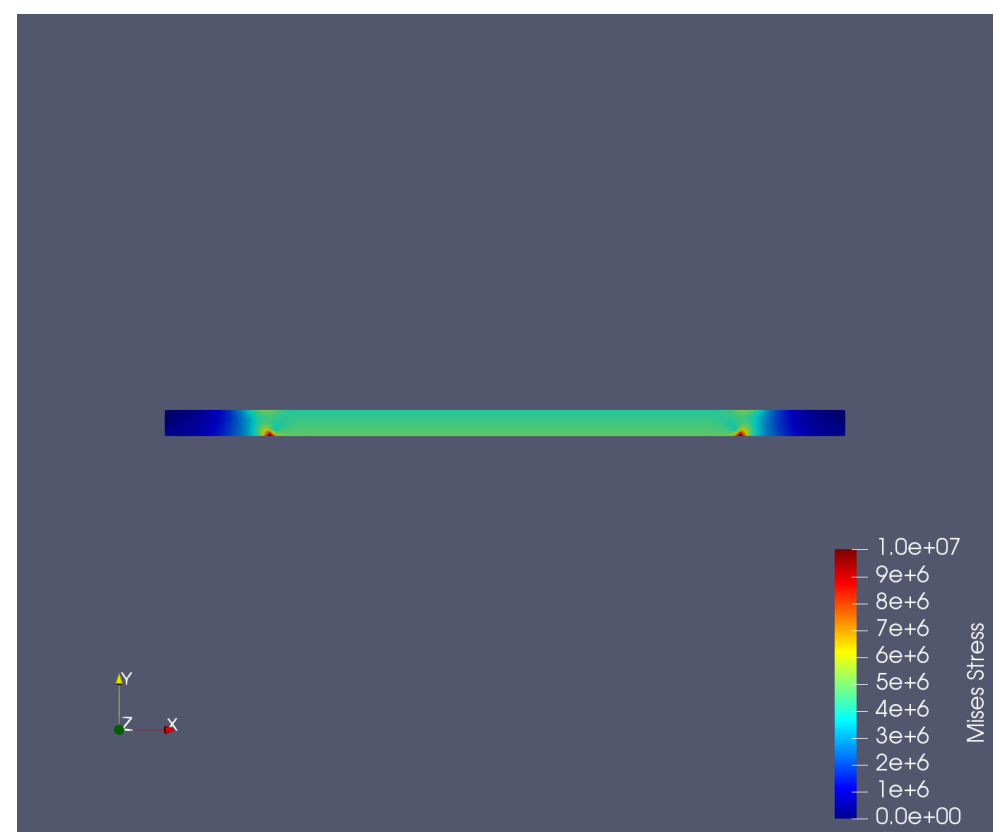

Supplemental Figure 12: Mises stress in the test film at mid-span (linear elastic simulation) at an opening strain of approximately 1.42 mstrain; cross-sectional view. Stress in Pascals. 


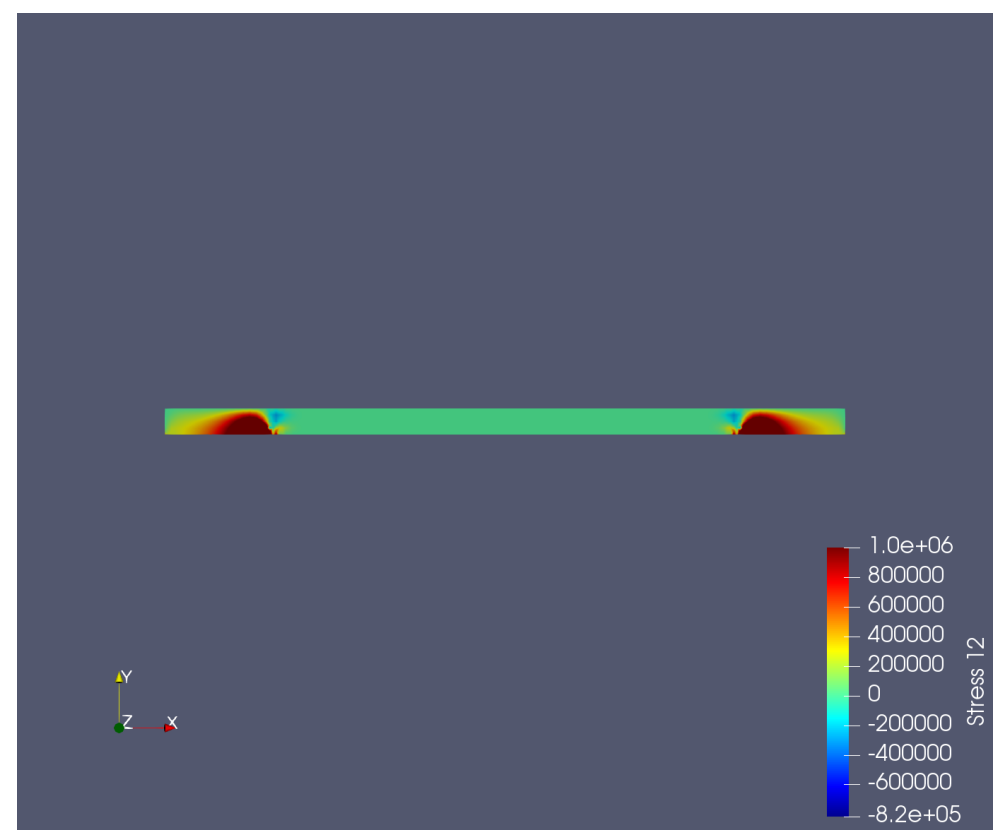

Supplemental Figure 13: Shear stress magnitude in the test film at mid-span (linear elastic simulation) at an opening strain of approximately 1.42 mstrain; cross-sectional view. Stress in Pascals.

\section{Shear stresses}

The $x y$-shear stress magnitude is shown in Supplemental Figure 13. This shows how the grips induce a shear stress in the film above the grips which is converted by the geometry into the tensile stress in the freestanding portion of the specimen. Note that the $x y$-shear stresses rapidly decrease in the freestanding portion of the film. Observe also the stress concentration at the edge of the grips. 


\section{Areal Chain Segment Density Calculation}

Using the analysis provided by Wool (1993) the molecular area, $a$, can be estimated using the volume-to-length ratio of the unit cell [3]:

$$
a=\sqrt{2} \frac{V}{L}
$$

The prefactor, $\sqrt{2}$, accounts for the random orientation of chains crossing the plane, and:

$$
\begin{aligned}
& V=\frac{z M_{0}}{\rho N_{A}} ; \quad L=c \text {-axis length (backbone direction) } \\
& \text { where: } \quad z=\text { number of monomers per c-axis length } \\
& M_{0}=\text { monomer molecular weight } \\
& N_{A}=\text { Avogadro's number }^{\prime} \\
& \rho=\text { density }
\end{aligned}
$$

The values for polystyrene were obtained from Table 1 in [3]:

$$
L=6.5 \AA ; \quad z=3 ; \quad M_{0}=104 \mathrm{~g} / \mathrm{mol}
$$

For the polystyrene used in this work: $\rho=1.04 \mathrm{~g} / \mathrm{cm}^{3}$

Which yields: $a=1.1 \times 10^{-14} \mathrm{~cm}^{2}$

The areal chain segment density is then: $p=\frac{1}{a} \Longrightarrow p=9.2 \times 10^{13}$ segments $/ \mathrm{cm}^{2}$ 


\section{Supplemental Video Captions}

Supplemental Video 1 - In situ video of PTP test, $240 \mathrm{~nm}$ thick polystyrene Supplemental Video 2 - In situ video of PTP test, $330 \mathrm{~nm}$ thick polystyrene Supplemental Video 3 - In situ video of PTP test, $250 \mathrm{~nm}$ thick polystyrene

*Note: The oscillations observed in the SI videos were due to vibrations in the laboratory during in situ testing with the optical microscope. The amplitude of these vibrations was small and the frequency fast enough such that they were not visible when viewed through the microscope. The oscillations seem slower in the video, but this is an artifact of the limited frame rate.

\section{References}

(1) Taylor, R. L.; Govindjee, S., FEAP - A Finite Element Analysis Program: Version 8.6 User Manual, 2020.

(2) Williams, M. L. ASME Journal of Applied Mechanics 1952, 19, 526-528.

(3) Wool, R. P. Macromolecules 1993, 26, 1564-1569. 